approximately one half, including anemia in 40\%. (Ganesan V et al. Ann Neurol 2003;53:167-173; Ped Neur Briefs Feb 2003;17:15) Other risk factors include activated protein $\mathrm{C}$ resistance, elevated lipoprotein(a), antiphospholipid antibodies, prothrombin gene variant, factor $\mathrm{V}$ Leiden mutation, subacute varicella infection, and methylenetetrahydrofolate reductase (MTHFR) mutation. IDA is a greater risk factor for sinovenous thrombosis than for arterial ischemic stroke.

\title{
NEONATAL SINOVENOUS THROMBOSIS AND MRI FINDINGS
}

Neonates with suspected sinovenous thrombosis were examined by magnetic resonance venography, diffusion-weighted imaging, and T2-weighted imaging, in a study at the Massachusetts General Hospital, Boston, MA. Of 200 CT scans performed in neonates 2004-2005, 15 neonates had intracranial hemorrhage or sinovenous signal suggesting sinovenous thrombosis. The mean birth weight was $3240 \mathrm{~g}$ and gestational age was 40 weeks. Presenting signs were seizures $(60 \%)$, apnea, hypotonia, and lethargy. Twelve patients had obstetric and perinatal complications; only 1 had sepsis. MRI showed a definite intraluminal clot in the deep venous system in 2 cases, and the remainder showed decreased flow-related enhancement within the dural venous sinuses. The sinus in all these cases was compressed by subdural hematoma or sutural diastasis. Parenchymal abnormalities were present in 5, classified as hemorrhage and cytotoxic edema in 3, or vasogenic edema in 1. Four of these 5 patients showed improvements, but one died. Intraluminal clot was rare in the superficial venous system. (Eichler F, Krishnamoorthy K, Grant PE. Magnetic resonance imaging evaluation of possible neonatal sinovenous thrombosis. Pediatr Neurol Nov 2007;37:317323). (Respond: Dr Grant, Department of Radiology, Massachusetts General Hospital, 55 Fruit St, Boston, MA 02114).

COMMENT. MRI with MR venography is recommended to assess for intraluminal clot and parenchymal injury when an initial CT scan shows a suspected sinus venous thrombosis. The characteristic MR appearance in venous thrombosis is loss of the normal flow enhancement on MR venography and corresponding filling defect on T2 weighted image. Clinical signs are not specific, and MRI is essential in diagnosis of neonatal sinovenous thrombosis. Deep venous thrombosis is often associated with intraventricular or thalamic hemorrhage. One third of term neonates with intraventricular hemorrhage have cerebral sinovenous thrombosis (Wu YW et al. Ann Neurol 2003;54:123-126). Obstetric complications were frequent in the above patients, and occipital bone molding may be associated with subdural or epidural hematoma leading to displacement of venous sinuses, venous hypertension and thrombosis.

\section{TOPIRAMATE COMPARED TO ACETAZOLAMIDE IN TREATMENT OF IDIOPATHIC INTRACRANIAL HYPERTENSION}

The efficacy of topiramate in the treatment of idiopathic intracranial hypertension (IIH) was compared to acetazolamide in an open-label study of 40 patients (age range 16-50, median 32 years; male/female ratio 5/35) at Ege University Medical School, Izmir, Turkey. Patients were assigned alternately to topiramate (100-150 mg daily) and acetazolamide (1000-1500 mg daily). CSF pressures (mmH20) ranged from 225-850, median 342 and 300, 
and were similar in the 2 groups at the beginning of the study. Visual fields compared at the end of the third, sixth, and twelfth month of the trial were significantly improved in both groups. Relief of headache occurred after a mean of 3.75 and 3.3 months treatment. Papilledema began to regress after the second month. Side effects of topiramate included distal paresthesias and concentration difficulties; acetazolamide caused fatigue and distal paresthesias. A prominent weight loss (mean $9.75 \mathrm{~kg}$ ) in the topiramate group was significantly greater than in acetazolamide treated patients $(p<0.001)$. Both drugs were equally effective in treatment of $\mathrm{IIH}$. A placebo-controlled, double-blind trial of topiramate in IIH is recommended. (Celebisoy N, Gokcay F, Sirin H, Akyurekli O. Treatment of idiopathic intracranial hypertension: topiramate vs acetazolamide, an open-label study. Acta Neurol Scand November 2007;1 16:322-327). (Respond: Dr Nese Celebisoy, Department of Neurology, Ege University Medical School, Bornova, Izmir, 35100, Turkey. E-mail: nese.celebisoy(ajege.edu.tr).

COMMENT. Acetazolamide is the first-line drug in treatment of IIH. It acts by inhibiting carbonic anhydrase and reducing CSF production. Topiramate also inhibits carbonic anhydrase, and the mechanism of action in IIH may be similar to that of acetazolamide. The above patient cohort included some adolescents, but the majority were adults. Children with IIH differ from adults in a slight preponderance of male vs female and a much smaller incidence of obesity. Of 10 prepubertal pediatric patients studied at Children's Hospital Los Angeles, only one was obese, and the most common presenting signs were stiff neck, diplopia and strabismus. Papilledema and sixth nerve palsy resolved rapidly with acetazolamide. (Cinciripini GS et al. Am J Ophthalmol 1999;127:178-182). A 14-year-old male adolescent with IIH and almost daily headache responded within 2 to 4 weeks following topiramate therapy. (Palacio E et al. Headache 2004;44:436-437). The results of the Turkish study are of interest, and a trial of topiramate in children with IIH is warranted.

\section{FAMILIAL BRAIN ARTERIOVENOUS MALFORMATIONS}

Researchers at University Medical Centre Utrecht, the Netherlands, reviewed the literature on patients with familial brain arteriovenous malformations (BAVMs), and their age, sex, and clinical presentation were compared with those in population-based patients with sporadic BAVMs. A total of 53 patients were identified in 25 families, including 3 families at the Utrecht center. Mean age at diagnosis of familial cases of BAVM was 27 years (range 9 months to 58 years), and 8 years younger than sporadic cases. Sex incidence and presenting symptoms were similar in familial and sporadic cases. Male: female ratio was $1: 1$, and presenting symptoms were intracranial hemorrhage in $47 \%$, epilepsy $26 \%$, headache $9 \%$, and focal neurologic deficit in $2 \%$. Clinical anticipation was likely in families with BAVMs in successive generations; children born to a parent with AVM presented at a mean age 22 years younger (17 vs 39 years). (van Beijnum J, van der Worp HB, Schippers HM et al. Familial occurrence of brain arteriovenous malformations: a systematic review. J Neurol Neurosurg Psychiatry November 2007;78:1213-1217). (Respond: Dr Janneke van Beijnum, Department of Neurology and Neurosurgery, University Medical Centre Utrecht, GO3. 124, PO Box 85500, 3508 GA Utrecht, the Netherlands. E-mail: J.vanBeijnum(a) umcutrecht.nl). 